\title{
Temporomandibular Disorder, facial pain and the need for high level information
}

\author{
Paulo César Rodrigues Conti \\ Associate Editor, Journal of Applied Oral Science - Department of Prosthodontics, Bauru School of Dentistry \\ Leonardo Rigoldi Bonjardim \\ Assistant Professor, Department of Biological Sciences, Bauru School of Dentistry
}

Dear Readers,

Temporomandibular disorders (TMD), a subclassification of musculoskeletal disorders, comprise a group of clinical problems affecting the masticatory muscles, the temporomandibular joint (TMJ), surrounding tissues components and combinations of these problems. These musculoskeletal disorders can produce signs and symptoms such as: functional limitation or deviation of the jaw during mandibular movements, joint sounds and pain in the TMJ and/or in the masticatory muscles ${ }^{2}$. Important features of this process are to be aggravated by jaw function, to present muscle and TMJ tenderness to palpation that reproduces the patient's chief complaint and to have a correspondence between the increased pressure applied and the pain report.

Pain related to TMD is a great clinical challenge in dentistry and represents a major cause of nondental pain in the orofacial region ${ }^{7}$. TMD is thought to have a multifactorial etiology, including physical ${ }^{6}$, genetic ${ }^{5}$ and psychosocial ${ }^{3}$ factors.

Regarding the evidence of associations between psychosocial factors and TMD, anxiety and depression have been extensively studied, however, little is known about the temporality between these conditions. To the best of our knowledge, a unique cohort study verified that depressive and anxiety symptoms should be considered as risk factors for TMD pain ${ }^{4}$.

In this sense, in the current issue the Journal of Applied Oral Science (JAOS) brings a study that investigates the association between anxiety/depression and TMD in a homogeneous population of college students. In the original study, authors evaluated Physical Therapy students and included those with diagnosis of TMD. These subjects were investigated two times about state of anxiety and depression, mandibular function, clinical signs and electromyographic activity of masticatory muscles. The authors describe a correlation between anxiety/ depression and some variables in subjects with TMD'.

Readers should be alert to the study of other psychosocial variables such as lifestyle, optimism (positive construct), catastrophizing (negative construct) that have been less studied in relation to TMD pain, although there is evidence of its influence on pain perception.

Additionally, the present issue of the journal brings out an overview of risk factors related to TMJ response to mandibular advancement surgery. Authors conducted a literature search in different databases, and according to selected studies, highlighted, among other aspects, that surgery was unable to influence TMJ with preexisting displaced discs and crepitus and that the rigidity of the fixation technique seemed to influence TMJ position and health. However, based on methodological problems found in many articles, more evidenced-based studies are required to understand the response of the TMJ after mandibular advancement surgery ${ }^{8}$.

These two contributions are important to collaborate for a better understanding of the puzzle related to TMD etiology and possible risk factors. TMD and related Orofacial Pain are challenging fields in contemporary dentistry, and our profession is encouraged to search for evidence-based information such as these two articles found in the current issue of the JAOS.

We hope you enjoy reading both papers and continue to disseminate the concept of evidence-based health care. In other words, high level information is needed to create a high level dental care. Our patients will be eternally greatful.

\section{REFERENCES}

1- Calixtre LB, Grüninger BLS, Chaves TC, Oliveira AB. Is there an association between anxiety/depression and temporomandibular disorders in college students? J Appl Oral Sci. 2014;22:15-21.

2- De Leeuw R. Dor orofacial: guia de avaliação, diagnóstico e tratamento. $4^{a}$ ed. São Paulo: Quintessence; 2010.

3- Fillingim RB, Ohrbach R, Greenspan JD, Knott C, Dubner R, Bair $E$, et al. Potential psychosocial risk factors for chronic TMD: descriptive data and empirically identified domains from the OPPERA case-control study. J Pain. 2011;12(11 Suppl):T46-60. 4- Kindler S, Samietz S, Houshmand M, Grabe HJ, Bernhardt O, Biffar $R$, et al. Depressive and anxiety symptoms as risk factors for temporomandibular joint pain: a prospective cohort study in the general population. J Pain. 2012;13(12):1188-97.

5- Oakley M, Vieira AR. The many faces of the genetics contribution to temporomandibular joint disorder. Orthod Craniofac Res. 2008;11:125-35.

6- Ohrbach R, Fillingim RB, Mulkey F, Gonzalez Y, Gordon S, Gremillion $\mathrm{H}$, et al. Clinical findings and pain symptoms as potential risk factors for chronic TMD: descriptive data and empirically identified domains from the OPPERA case-control study. J Pain. 2011;12(11 Suppl):T27-45

7- Okeson JP, de Leeuw R. Differential diagnosis of temporomandibular disorders and other orofacial pain disorders. Dent Clin North Am. 2011;55(1):105-20.

8- Valladares-Neto J,Cevidanes LH, Rocha WC, Almeida GA, Paiva JB, Rino-Neto J. TMJ response to mandibular advancement surgery: an overview of risk factors. J Appl Oral Sci. 2014;22:2-14. 